\title{
Experimental Verification of Anomalous Forces on Shielded Symmetrical Capacitors
}

\author{
Elio B. Porcelli ${ }^{1}$, Omar R. Alves ${ }^{1} \&$ Victo S. Filho ${ }^{1}$ \\ ${ }^{1}$ H4D Scientific Research Laboratory, 04674-225, São Paulo, SP, Brazil \\ Correspondence: Elio B. Porcelli, H4D Scientific Research Laboratory, 04674-225, São Paulo, SP, Brazil. \\ E-mail: elioporcelli@h4dscientific.com \\ Received: November 12, 2019 \\ Accepted: March 25, 2020 \\ Online Published: March 30, 2020 \\ doi:10.5539/apr.v12n2p33 \\ URL: https://doi.org/10.5539/apr.v12n2p33
}

\begin{abstract}
In this work, we measured the magnitude of forces raised from the operation of symmetrical capacitor devices working in high electric potentials. Our experimental measurements were realized with basis on an improved setup which aimed significant reduction of ionic wind by means of an efficient shield. We observed small variations of the device inertia within an accurate range and we confirmed with good accuracy that the experimental results can be explained by a generalized quantum entanglement hypothesis which provides us a theoretical model for a macroscopic dipole force raised by the myriad of microscopic dipoles constituting the capacitor. The new results corroborated the positive results of previous experiments and also indicate the validity of our theoretical forecast.
\end{abstract}

Keywords: high-energy capacitors, dipole forces, dielectric breakdown, quantum entanglement, anomalous weight reduction

\section{Introduction}

\subsection{Outline of the Phenomenon}

It is well known since 1928 (Brown, 1928, 1965; Williams, 1983) that an anomalous phenomenon involving weight variation measurements occurs in experiments in which parallel plate capacitor devices are subjected to high voltage. Such an anomaly known in some works as Biefeld-Brown effect is characterized by a reduction on their weight or the presence of a very small anomalous net upward force which appears on capacitors when subjected to high-voltage. Since then many works have investigated the phenomenon in order to confirm its existence and determine its origin (Buehler, 2004; Mahood, 1999; Modanese,2013; Musha, 2008; Poher \& Poher, 2011; Woodward, 1991, 1992, 1996). In Antano (2012), it was reported that trials to explain the phenomenon were realized, mainly by means of the hypothesis of the existence of an electric wind (Christensen Moller, 1967; Einat, \& Kalderon, 2014; Tajmar, 2004; Ianconescu, Sohar, \& Mudrik, 2011). However, measurements performed in the vacuum showed that the effect persisted even so (Antano, 2012; Brown, 1965). Besides, other experiments described in Refs (Buehler, 2004; Musha, 2008; Musha, 2014) showed that insulating materials around the capacitor devices could not eliminate such forces. Further, they could not be explained from the electromagnetic interaction or fields of the Earth due to the inconsistency with the magnitude of the upward force.

Such physical systems involving symmetrical capacitors were also previously investigated in a systematical way from their operation under high voltages (Porcelli \& Filho, 2015, 2016) in order to analyze the existence of the effect from a huge quantity of data. It was experimentally verified the presence of anomalous forces by implementing an innovation on the experimental procedure which consisted in collecting hundreds of force data from an advanced accelerometer. It was also proposed a theoretical framework which was able to explain the experimental results (Porcelli \& Filho, 2015, 2016) as also of other authors (Buehler, 2004; Mahood, 1999; Musha, 2008; Poher \& Poher, 2011; Musha, 2014). The procedure proposed considered two states: (i) a state in which the power source was turned off and the accelerometer measured the values of acceleration; and (ii) the state in which the power source was turned on and the same measurements were implemented. The difference between the values of acceleration of the states on and off corresponded to the average net force acting on the capacitor devices.

However, despite of positive results there could be a negligible possibility that the forces were originated from ionic wind effect, although the magnitude of ionic wind forces were extremely smaller than the values measured, according to Musha (2008). So, our motivation in the present paper was to investigate if the ionic wind effect could be responsible for the anomaly. In order to clarify that important and specific point we conceive and implement an improved experimental setup 
with a more efficient shield, so that we could detect possible significant differences between the forces measured with and without shielding and determine the probable nature of such forces.

In next sections, this work describes our improved experimental setup and reports the new measurements of force obtained from that configuration. In the following, we describe in a more detailed way the experiments previously performed, detail our experimental setup, as well our measurements concerning to weight variations of the capacitor devices. Afterwards, we present our theoretical model in order to present an explanation of the phenomenon. Finally, in the last section we discuss our conclusions and final remarks.

\section{Method}

\subsection{Previous Experimental Works}

In order to understand the controversy between two currents of research, we verified that many authors reported the existence of the anomalous forces in capacitors, but some of them asserted that it could be due to ionic wind or would not even exist. For instance, Mahood (1999) reports many measurements of inertia in capacitors operating under high voltage which indicate small weight reduction. Einat and Kalderon (2014) reports the existence of forces in asymmetrical capacitors and as higher the asymmetry as stronger is the magnitude of the force (with a enhancement factor which can achieve 9). Other experimental works described in Refs (Woodward, 1991, 1992, 1996) indicated quick oscillations of the energy density of high-energy capacitors which corresponded to reduction in weight of up to $10 \%$. Even so, the effect remains controversial, mainly due to the weakness of the force generated in the experimental process. For instance, in Tajmar (2004) it was reported that the effect would not exist and it would be caused only due to ionic wind. The same conclusion is reported in Ianconescu Sohar and Mudrik (2011), being proposed a calculation of the levitation force due to ion wind. In an opposite conclusion, the laboratory Honda also analyzed the phenomenon in Refs. (Woodward, 1991, 2014) in a lot of experiments performed to specifically verify the Biefeld-Brown effect. The experimental setups were composed by rounded symmetrical parallel capacitor devices in order to verify if the corona discharges and electric wind around the capacitor could explain the effect. So, they applied AC and DC voltages up to $18 \mathrm{kV}$ on capacitors of materials such as glass and plastic with different thicknesses, some of them enclosed by an insulator oil contained within a metallic vessel. The measurements indicated weight losses within $(0.28,1.52) \mathrm{g}$. However, the forces generated by the ionic transfer of momentum, feature needed for the electric wind hypothesis, were reported in Musha (2008) and indicated inertia reduction of the order of $0.001 \mathrm{~g}$ due to ionic wind. In other words, such an explanation would not be possible because the value represented orders of magnitude less than the value of the weight losses measured in that work.

Although such a controversy and disagreement persists, a high number of works have been studying the phenomenon, including more recent works (Porcelli \& Filho, 2015, 2016a, 2016b, 2017) based on a model of dipole forces. The effect still needs more investigation, accurate experimental verification and a good theoretical explanation, as proposed in detail in Porcelli and Filho (2017), based on the theoretical framework of Generalized Quantum Entanglement (GQE). We analyze our experiments by considering such a theoretical framework in the present work, which allows us in a simple way to empirically define the dipole force on capacitors under high voltage. We summarize such an empirical model in section 3 .

In Porcelli and Filho (2015, 2016a), some experimental measurements were initially performed with the objective of verifying the maximum weight loss of a high-potential symmetrical capacitor sample by considering a capacitor sample of two parallel rounded plates made by aluminium and $8 \mathrm{~cm}$ of diameter, enclosing a plastic dielectric (polystyrene) $1 \mathrm{~mm}$ tick, with mass $41.154 \mathrm{~g}$ and relative permittivity 2.7. The maximum average weight loss was up to $220 \mathrm{mgf}$ for a maximum DC voltage of $20 \mathrm{kV}$. The measurement of the capacitor weight was made by a milligram electronic scale of $300 \mathrm{~g}$ maximum load. Next it was considered the procedure previously described of using an advanced accelerometer to measure variations of weight of the device, by collecting a huge quantity of force data and concluding from them which the results were positive for the presence of small upward forces on the devices for some values of voltage applied in the setup implemented in the experiments.

In the present work, we adopted and it was enough to consider the initial method, simply using the electronic scale, trusting that the setup conceived in the experiment could allow us to obtain the measurements with less oscillations. We describe that improved setup and the experimental work in next section.

\subsection{Improved Experimental Setup}

In order to improve the previous experimental setup, we implemented in the present work an experimental apparatus in which some components of the circuit were insulated from electromagnetic interference and the sample of capacitor was shielded so that it was not significantly subjected to ionic wind at all, by inserting the device inside a blinding camera (or box). So, there was not enough air inside the system so that the ionic wind effect could achieve significant influence on the results, although it could exist yet. The setup really improved the analysis of the magnitude of the force acting 
on the capacitors without the possible significant influence of the ionic wind effect. All insulated wires for supplying the capacitor with high voltage were shielded accordingly and their metallic coverage was connected in the same laboratory electrical potential with the capacitor box coverage in order to eliminate any electrostatic interaction with the environment around.

The Figure 1 shows us the diagrammatic scheme of the circuit implemented in the experiments and in the Figure 2 we see a photo of the experimental setup built to realize the measurements of anomalous forces for high values of the voltage applied. The equipments as the instruments of measurement and the capacitor sample, including their technical features, are indicated in the Table 1.

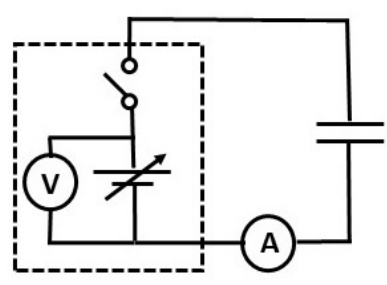

Figure 1: Scheme of the experimental setup built to the measurements of the weight losses of symmetrical capacitors. The high-voltage power supply showed inside the dashed area includes a DC voltage generator, volt meter and on-off switch. The power supply provides the DC high voltage to the symmetric capacitor where the ampere meter is connected in serial to monitor the electric current.

Table 1: List of devices used in the experiment (sample and instruments of measurements) with correspondent technical details.

\begin{tabular}{c} 
Devices \\
Multimeter UNI-T model UT30B \\
High Voltage Power Supply with Voltmeter - 0 40 kV DC \\
Milligram Digital Scale C BEL model \\
Circular Symmetric Capacitor C 2mm PMMA dielectric thickness C 69 mm radius \\
\hline
\end{tabular}

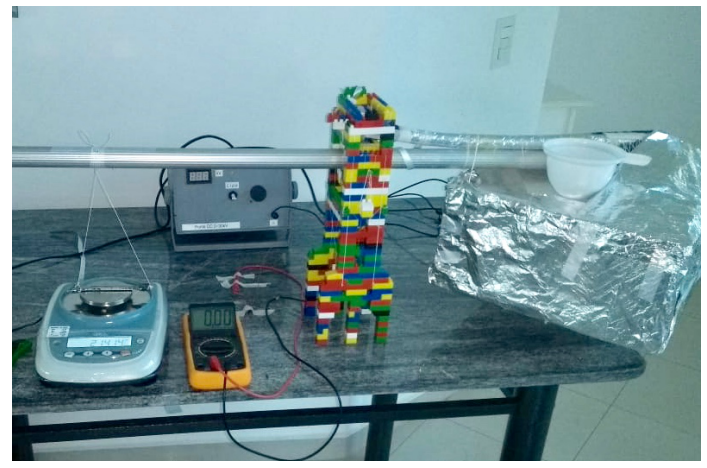

Figure 2: Photo of the experimental setup implemented to realize the measurements of anomalous upward forces acting on the symmetric capacitor. The equipments used in the experiment were a milligram digital scale, an ampere meter, the high-voltage power supply with volt meter (in the background), pivot (built with Lego) and box with aluminum coverage. A metallic arm supported by the pivot connects mechanically the box where the symmetric capacitor is enclosed and the counterweight in its ends. 
The photo shows the equipments used in the experiment such as milligram digital scale, ampere meter, high voltage power supply with volt meter, pivot (built with plastic blocks) and box with aluminum coverage. A metallic arm supported by the pivot connects mechanically the box where the symmetric capacitor is enclosed and the counterweight in its ends. Two small bearings were used on the pivoted shaft of the scale. The bearings coupled to the shaft supported by the pivot allowed to significantly decrease the friction in order to improve the motion of the arm. In the experiment, we monitored the current via ampere meter in order to avoid some possible current leakage and also the dielectric breakdown.

In our experimental work, we implemented the same initial procedure for the measurements as first described in Porcelli and Filho (2015), from which one adopts the electronic scale to measure the variations on the weight of the capacitor.

We applied an initial voltage on our sample capacitor and slowly varied the tension from $5 \mathrm{KV}$ up to nearly $25 \mathrm{kV}$, measuring the average weight reduction in $\mathrm{mg}$ (or $\mathrm{mgf}$ ) for some values of the voltage applied on the capacitor. The anomalous force was detected in all of high values of voltage and the average dependence of that force on the square of the voltage applied is well determined, as one can see in details in section 4.

In other words, by following the procedure described, we obtained measurements that are consistent with the weight loss hypothesis, mainly for the higher voltages applied.

In terms of possible future applications, the weakness of the effect would be a restriction, but in hypothesis the effect could be enhanced if we used a device with higher physical values as the capacitance. In fact, in literature was already reported supercapacitance (Markoulidis, Lei, \& Lekakou, 2013; Dufour, Dufour, Dioury, \& Vinko, 2017), capacitances optically controlled through a dielectric constant (Yamasaki et al., 2003) or supercapacitor cells made of MWCNTS/nickel oxide nanocomposite (Adekunle, 2011), as some of possible examples to investigate.

We now present in the next section the theoretical proposal in order to explain the effect. Provided by such a theoretical proposal, we could explain our experimental results.

\section{Theoretical Description}

\subsection{Generalized Quantum Entanglement}

As described in our earlier works (Porcelli \& Filho, 2015, 2016a, 2016b, 2016, 2017; Porcelli, 2015; Porcelli \& Filho, 2017) the theoretical framework that leads us to a macroscopic description of our systems is based on the Generalized Quantum Entanglement (GQE) hypothesis. As already known from literature (Zeilinger, 1999), the quantum entanglement is a nonlocal phenomenon and the consequence of an interaction in the past between two or more particles in a given time, so that afterwards all of particles in the quantum state maintain the information from each other, even considering huge distances (Zeilinger, 1999). The possible effects of GQE in physical systems of large quantity of particles is a relevant subject to be studied if we want to understand and determine its macroscopic influence. As the theoretical analysis is too much complicated, it is natural to avoid the problem of analyzing if all the particles in the universe could be entangled among them. However, some researchers have lately given attention to the problem, as reported in a recent work (Buniy \& Hsu, 2012).

Although there is high complexity in the quantum problem of many entangled particles, some macroscopic observables can be determined from the microscopic behavior by means of a connection between the micro and macro world, so that we can successfully calculate their physical values in an easier way and relatively good accuracy, as described in the formalism reported in details in Porcelli and Filho (2017). Rather by assuming that in extreme conditions there could be the unusual occurrence of quantum entanglement macroscopic effects, it could hypothetically be revealed by supposing that the microscopic particles of our system can collectively have influence on the magnitude of some macroscopic observables describing the physical system. So, we assume preexisting quantum entanglement and its influence on the macroscopic observables of the systems, as already checked in our previous physical systems studied, constituted by electric dipoles or by photons and electrons. Our present physical system can be analyzed as a possible case of application by means of our GQE hypothesis. In fact, our conjecture has already been accurately applied in many physical systems, as in the cases of anomalous forces in the operation of symmetrical (Porcelli \& Filho, 2015, 2016a) or asymmetrical capacitors (Porcelli $\&$ Filho, 2016b), magnetic cores (Porcelli \& Filho, 2017), piezoelectric materials (Porcelli, 2015) or semiconductor laser diodes (Porcelli \& Filho, 2017). As an example, for capacitors it is needed to apply a high voltage in order to realize the presence and the influence of GQE among the dipoles of the dielectric and the external environment.

Basically, the method of calculation considers that in some physical conditions the generalized entanglement among the microscopic constituents of a physical system - or the property of preexisting state of generalized quantum entanglement - can exist, manifest itself in extreme conditions and its effects could be detected in macroscopic scale and also can be calculated, in order to obtain the values of macroscopic observables associated to such systems.

Such ideas concerning to quantum entanglement for many bodies or quantum information in macroscopic scale have 
been more and more studied in some works (Amico, Fazio, Osterlosh, \& Vedral, 2008; Vedral, 2010, 2011; Ghosh, Rosenbaum, Aeppli, \& Coppersmith, 2003; Wiesniak, Vedral, \& Brukner, 2005), including the analysis of the dynamics of the measurement-induced nonlocality of two-qubit states undergoing decoherence induced by a structured environment (Ma, Cheng, \& Ying, 2017). The dynamical behavior of quantum correlations have been a topic of intense interest due to its application to quantum information. For instance, in Franco et al. (2013), correlations with no classical equivalence as quantum entanglement or nonlocal correlations are analyzed in open quantum systems for bipartite systems embedded in non-Markovian environments. Besides, it is reviewed the phenomenon of entanglement revivals in a two-qubit system for both independent environments and a common environment. In a different context, there are some important works (Wu, Bandyopadhyay, Sarandy,\& Lidar, 2005; Toth, 2005; Dowling, Doherty, \& Bartlett, 2004; Hao \& Zhu, 2007; Chakraborty, Singh, Das, Sen, \& Mitra, 2012; Wei, Kais, \& Chen, 2010) that show the existence of the so-called quantum witness, that is, the existence of a macroscopic observable revealing quantum entanglement between individual spins in a solid. In particular, in Vedral (2011) was shown that the quantum entanglement can explain a lot of macroscopic properties in high temperatures. It is also relevant to say that such a feature of manifestations of quantum entanglement for many particles in the macroscopic level (Amico, Fazio, Osterlosh, \& Vedral, 2008; Vedral, 2010, 2011; Ghosh, Rosenbaum, Aeppli, \& Coppersmith, 2003) allows us to use the classical formulation in our calculations due to the inequality relating local and nonlocal quantities in Wiesniak, Vedral, and Brukner (2005). As reported in Wiesniak, Vedral, and Brukner (2005), the entanglement witness is a more general phenomenon, in the sense that it is not only valid for special materials, associating some macroscopic observables such as magnetic susceptibility with spin entanglement between individual constituents of a solid. So, there is a relation between the physical quantities, that is, a macroscopic quantum complementary relation between magnetization $M$, representing local properties, and magnetic susceptibility representing nonlocal properties. If we considere $N$ as the number of spins for a system of an arbitrary spin length $s$ in a lattice, we can write such a relation by defining

$$
Q_{n l}=1-\frac{k T \bar{\chi}}{N s}
$$

and

$$
Q_{l}=\frac{\langle\vec{M}\rangle^{2}}{N^{2} s^{2}}
$$

in which $\mathbf{M}$ is the magnetization vector, $k$ is the Boltzmann constant, $T$ the temperature and the total susceptibility (with bar) is defined as the sum of the susceptibilities along the three spatial directions $\mathrm{x}, \mathrm{y}$ and $\mathrm{z}$. Then it was shown in Vedral (2011) that one can write:

$$
Q_{n l}+Q_{l} \leq 1
$$

Such quantities have specific meanings, that is, $Q_{n l}$ represents the quantum correlations between the spins in the solid (nonlocal properties) and $Q_{l}$ represents the local properties of individual spins.

\subsection{Model for the Symmetrical Capacitor}

From the theoretical framework described, we now present the theoretical model for our case involving the shielded highenergy capacitor device by considering the concrete way of deterministic calculation by means of macroscopic quantities. In Porcelli and Filho $(2015,2016)$, it was defined the electric dipole force $F$ which is generated on a capacitor due to the microscopic forces raised by the myriad of electric dipoles constituting the material. According to our empirical analysis, conceived from GQE and the concept of quantum witness (Wiesniak, Vedral, \& Brukner, 2005) such a force is given by the relation

$$
F=\frac{0.102}{16 . \pi^{2}} \frac{\epsilon_{r}-1}{\epsilon_{r}+2} \epsilon_{0} A E^{2},
$$


in which the force $F$ is in units of $\mathrm{Kgf}, A$ is the area of the capacitor plate, $E$ is the electric field applied in the medium and the subscripts $r$ and 0 indicate the relative permittivity of the material and the dielectric constant of the vacuum.

Since the dielectric material (PMMA) is a plastic, we performed the same calculation criteria shown in our previous works (Porcelli \& Filho, 2015, 2016) in which we also use plastic dielectrics so that we get the relative permittivity 1.086.

We compare the measurements of weight losses of all the experiments reported in this work and the theoretical forecast based on our dipole force model.

Our experiments also showed that the weight losses (in mgf) increased for higher voltage applied (in $\mathrm{kV}$ ) to the plates of the symmetrical capacitors. Further the effects occurs in mgf scale and the theory is very consistent with such an order of magnitude for the weight losses.

\section{Results}

As in Porcelli and Filho (2015), our experiments also showed that weight losses were verified in the scale of mgf and increased for higher voltage applied (in $\mathrm{kV}$ ) to the plates of the symmetrical capacitors. The Table 2 compares the values measured of weight variation with the correspondent theoretical values for each voltage applied on the capacitor.

Table 2: Measurements of weight variation of the capacitor (in mgf) versus the theoretical values for each voltage applied. The measurements of weight variation presented a $20 \%$ error.

\begin{tabular}{ccc} 
Voltage (V) & Theoretical (mgf) & Measured (mgf) \\
& & \\
\hline 5000 & 14.89 & 12.3 \\
7500 & 33.51 & 25 \\
10000 & 59.57 & 64 \\
15000 & 134.02 & 100 \\
20000 & 238.26 & 196 \\
25000 & 372.29 & 324 \\
& & \\
\hline
\end{tabular}

The measurements indicated that the anomalous force or weight variation has an average dependence on the square of the voltage applied on the capacitor. In Figure 3 the plot of the square root of the capacitor weight variation versus the voltage applied on the capacitor.

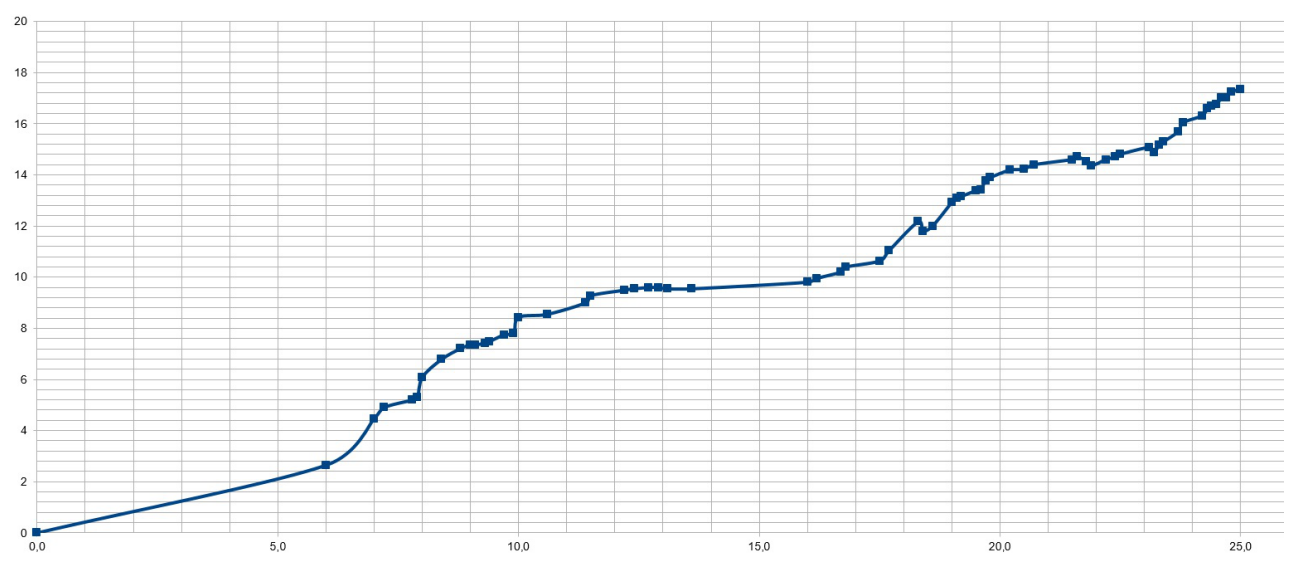

Figure 3: Plot of the square root of the capacitor weight variation (in $\mathrm{mgf}^{1 / 2}$ ) versus the voltage applied $(\mathrm{kV})$. The average dependence can be described by a straight line. 
The graphic in the Figure 3 was drawn with the square root of the variation of the capacitor weight in the vertical axis and the voltage applied in the horizontal axis in order to better observe the variation of curve (anamorphosis). The phenomenon can be associated to the variation of the dielectric constant when the frequency increases, as reported in the case of $\mathrm{TiO}_{2}$ (Mangrola, Joshi, Parmar, \& Pillai, 2013). In fact, this oscillation observed in the curve deserves more investigation but the experimental range of voltage values from $13 \mathrm{kV}$ to $25 \mathrm{kV}$ are a little below the theoretical ones possibly because of the usual (Murata Innovator in Electronics, n.d.; Maxim Integrated Tutorials, n.d., Vishay Sprague Report, n.d.) reduction of the capacitance according to the voltage applied increasing for an arbitrary dielectric substance.

It is relevant to emphasize that in the experiments reported for this improved setup in relation to the previous setups described in Refs. (Porcelli \& Filho, 2015, 2016a, 2016b) allowed us to decrease much more the uncertainty related to origin of the effect. In fact, due to the improved shield, the insulation and shielding, the possibility of electrostatic interaction of the capacitor with the environment is discarded and known interactions (as the electromagnetic one) could not be responsible for raising the phenomenon. In relation to the ionic wind, it is very reduced or even eliminated in the experimental setup elaborated because the capacitor was confined in an electrostatically shielded box connected to the ground of the laboratory and with no direct contact with air in the environment. Hence, it was reinforced that such a new setup suffers the action of the anomalous variation of the capacitor weight due to a new mechanism and, as we could verify in last section, it can be consistently explained by our theoretical model. In fact, the effect occurs in mgf scale and the theory is consistent with such an order of magnitude for the weight losses. The theoretical values calculated with basis on our theoretical framework for some values of voltage (order of $\mathrm{kV}$ ) were close to the anomalous forces experimentally measured, by considering errors around $20 \%$.

Despite of the weakness of the effect, it is worth to emphasize that the enhancement of the effect can allow the viability of power generation systems without fuels, as electromagnetic drives, so that one can apply them to aerospace vehicles. So, the study of such an effect can produce valuable applications in a recent future.

\section{Discussion}

In this work, we present an experimental investigation of the existence and magnitude of anomalous forces on shielded symmetrical capacitors, operating in high voltage. The motivation to perform such measurements was to implement an experimental setup with significant improvement in relation to a previous one, mainly characterized by an efficient camera for shielding any electromagnetic influence and with very restricted presence of air in contact with the capacitor.

Our experimental measurements indicated the presence of small but persistent weight losses or an upward force acting on the capacitor in the scale of mgf for different values of the high voltage applied on the capacitors. Our improved setup allowed us a higher shield of the capacitor when placed within the box and hence we could improve the accuracy of the measurements, so that less instabilities on the electronic scale occurred and we obtained more confidence that the effect was not due to the ionic wind effect. Our experimental results confirm the presence of a upward force acting on the system for different values of voltage.

We also propose a theoretical model in the macroscopic level of a dipole force, by considering the validity of the concepts of quantum witness and generalized quantum entanglement. By means of such a theoretical proposal we show that one can explain the manifestation of the effects caused by the microscopic dipoles on the macroscopic medium when the capacitor was subjected to high voltage. In fact, the theoretical results of the anomalous upward dipole force present good agreement with our experimental measurements of weight losses of the symmetrical capacitor sample by means of an electronic scale. The magnitude of the anomalous force seems to be directly related to the dipole force calculated and its nature is deeply related to the collective action of the internal electric dipoles from the dielectrics of symmetric capacitors working in high voltages, as figured out by GQE formalism.

We are now elaborating other work based on new experimental setups involving the enhancement of the effect and the detection of higher magnitudes of the anomalous forces and the effect of induction of forces at distance by the capacitor. As examples of application, such a effect can be applied in electromagnetic drives for the substitution of engines based on fuel in order to produce a thrust intense enough to move a spacecraft through the space.

\section{References}

Adekunle, A. S. (2011). Supercapacitive Properties of Symmetry and the Asymmetry Two Electrode Coin Type Supercapacitor Cells Made from MWCNTS/Nickel Oxide Nanocomposite. Int. J. Electrochem. Sci., 6, 4760-4774.

Amico, L., Fazio, R., Osterlosh, A., \& Vedral, V. (2008). Entanglement in Many-Body Systems. Rev. Mod. Phys., 80, 517 (2008). doi: 10.1103/RevModPhys.80.517

Antano, M. A. (2012). Biefeld-Brown Effect and Space Curvature of Electromagnetic Field, arXiv:1004.0810v2.

Brown, T. T. (1928). A Method of and an Apparatus or Machine for Producing Force or Motion. U.K. Patent No. 00.311. 
Brown, T. T. (1965). Electrokinetic Apparatus. U.S. Patent 3.187.206, 1965.

Buehler, D. R. (2004). Exploratory Research on the Phenomenon of the Movement of High Voltage Capacitors. Journal of Space Mixing, 2, 1-22.

Buniy, R. V., \& Hsu, S. D. H. (2012). Everything is Entangled. Phys. Lett. B, Vol. 718, 233-236. doi: 10.1016/j.physletb.20 12.09.047

Chakraborty, T., Singh, H., Das, D., Sen, T. K., \& Mitra, C. (2012). Quantification of Entanglement from Magnetic Susceptibility for a Heisenberg Spin 1/2 System. Phys. Lett. A, 376, 2967-2971. doi: 10.1016/j.physleta.2012.08.051

Christensen, E. A., \& Møller, P. S. (1967). Ion-Neutral Propulsion in Atmospheric Media. AIAA J., 5(10), $1768 C 1773$. doi: $10.2514 / 3.4302$

Dowling, M. R., Doherty, A. C., \& Bartlett, S. D. (2004). Energy as an Entanglement Witness for Quantum Many-Body Systems. Phys. Rev. A, 70, 062113. doi: 10.1103/PhysRevA.70.062113

Dufour, J., Dufour, X., Dioury, F., \& Vinko, J. D. (2017). Measurement of the Enthalpy of Formation of an Iron PicoHydride and of its Main Properties. Int. J. Mod. Phys. B, 31(25), 1745007. doi: 10.1142/S0217979217450072

Einat, M., \& Kalderon, R. (2014). High efficiency Lifter based on the Biefeld-Brown effect. AIP Advances, 4, 077120 1-20. doi: $10.1063 / 1.4890353$

Franco, R. L., Bellomo, B., Maniscalco, S., \& Compagno, G. (2013). Dynamics of Quantum Correlations in Two-Qubit Systems within Non-Markovian Environments. Int. J. Mod. Phys. B, 27, No. 01n03, 1345053. doi: 10.1142/S0217979213450537

Ghosh, S., Rosenbaum, T. F., Aeppli, G., \& Coppersmith, S. N. (2003). Entangled Quantum State of Magnetic Dipoles. Nature, 425, 48-51. doi: 10.1038/nature01888

Hao, X., \& Zhu, S. (2007). Entanglement in a Quantum Mixed-Spin Chain. Phys. Lett. A, 366, 206-210. doi: $10.1016 /$ j.physleta.2007.01.053

Ianconescu, R., Sohar, D., \& Mudrik, M. (2011). An analysis of the Brown-Biefeld effect. Journal of Electrostatics, 69, 512-521. doi: 10.1016/j.elstat.2011.07.004

Ma, X. S., Cheng, M.-T., \& Ying, Q. (2017). Dynamics of measurement-induced nonlocality of quantum states in a structured environment. Int. J. Mod. Phys. B, Vol. 31(2), 1650260. doi: 10.1142/S021797921650260X

Mahood, T. L. (1999). Propellantless Propulsion: Recent Experimental Results Exploiting Transient Mass Modification. In Mohamed S. el-Genk (Ed.), CP458 Space Technology and Applications International Forum.

Mangrola, M. H., Joshi, V. G., Parmar, B. H., \& Pillai, A. S. (2013). Optical and Dielectric Properties of the Cobalt Doped TiO2 Nanoparticles. Int. J. Mod. Phys.: Conf. Series, 22, 332-335. doi: 10.1142/S2010194513010313

Markoulidis, F., Lei, C., \& Lekakou, C. (2013). Fabrication of High-Performance Supercapacitors Based on Transversely Oriented Carbon Nanotubes. Appl. Phys. A, 111, 227-236. doi: 10.1007/s00339-012-7471-8

Maxim Integrated Tutorials. (n.d.). Retrieved from https://www.maximintegrated.com/en/app-notes/index.mvp/id/5527

Modanese, G. (2013). A comparison between the YBCO discharge experiments by E. Podkletnov and C. Poher, and their theoretical interpretations, arXiv: 1312.0958v1.

Murata Innovator in Electronics. (n.d.). Retrieved from https://www.murata.com/support/faqs/ products/capacitor/mlcc/ch ar/0005

Musha, T. (2008). Explanation of dynamical Biefeld-Brown Effect from the standpoint of ZPF field. JBIS, 61, $379-384$.

Musha, T. (2014). Theoretical explanation of the Biefeld-Brown Effect. Retrieved from http://www.ovaltech.ca/electrogra vity.html

Poher, C., \& Poher, D. (2011). Physical Phenomena Observed during Strong Electric Discharges into Layered Y123 Superconducting Devices at 77 K. Appl. Phys. Res., 3(2), 51-66. doi: 10.5539/apr.v3n2p51

Porcelli, E. B. (2015). Induction of force performed by the piezoelectric materials. US Patent US2015/0188026 A1.

Porcelli, E. B., \& Filho, V. S. (2015). On the Anomalous Weight Losses in High-Voltage Symmetrical Capacitors. arXiv:1502.06915.

Porcelli, E. B., \& Filho, V. S. (2016a). On the Anomalous Forces in High-Voltage Symmetrical Capacitors. Phys. Ess., 29, 2-9. doi: 10.4006/0836-1398-29.1.002 
Porcelli, E. B., \& Filho, V. S. (2016b). Characterisation of Anomalous Asymmetric High-Voltage Capacitors. IET Sci., Meas. E Tech., 10(4), 383-388. doi: 10.1049/iet-smt.2015.0250

Porcelli, E. B., \& Filho, V. S. (2017) Induction of Forces at Distance Performed by Semiconductor Laser Diodes. Am. J. Eng. Res. (AJER), Vol. 6(5), 35-48. ISSN Print: 2327-588X

Porcelli, E. B., \& Filho, V. S. (2017). Anomalous Effects from Dipole Environment Quantum Entanglement. Int. J. Adv. Eng. Res. Sci., 4(1), 131-144. doi: 10.22161/ijaers.4.1.21

Porcelli, E. B., \& Filho, V. S. (2018). Induction of force performed by piezoelectric materials. J. Power Eng. En. 6(1), 33-50. doi: 10.4236/jpee.2018.61004

Tajmar, M. (2004). Biefeld-Brown Effect: Misinterpretation of Corona Wind Phenomena. AIAA J., 42(2), 315-318. doi: $10.2514 / 1.9095$

Toth, G. (2005). Entanglement Witnesses in Spin Models. Phys. Rev. A, 71, 010301(R). doi: 10.1103/PhysRevA.71.010301

Vedral, V. (2010). Decoding Reality: the Universe as Quantum Information. Oxford University Press.

Vedral, V. (2011). A Vida em um Mundo Quântico. Sci. Am. Br., 110, 30.

Vishay Sprague Report. (n.d.). Retrieved from https://www.vishay.com/docs/40144/capchange.pdf

Wei, Q., Kais, S., \& Chen, Y. P. (2010). Entanglement Switch for Dipole Arrays. J. Chem. Phys., Vol. 132, 121104. doi: $10.1063 / 1.3366522$

Wieśniak, M., Vedral, V., \& Brukner, C. (2005). Magnetic Susceptibility as a Macroscopic Entanglement Witness. New J. Phys., 7, 258 1-8. doi: 10.1088/1367-2630/7/1/258

Williams, P. E. (1983). Possible Unifying Effect of the Dynamic Theory. Rep. LA-9623-MS Los Alamos Scientific Lab., Los Alamos NM.

Woodward, J. F. (1991). Measurements of a Machian Transient Mass Fluctuation. Found. Phys. Lett., 4, 407-423. doi: 10.1007/BF00691187

Woodward, J. F. (1992). A Stationary Apparent Weight Shift From a Transient Machian Mass Fluctuation. Found. Phys. Lett., 5, 425-442. doi: 10.1007/BF00690424

Woodward, J. F. (1996). A Laboratory Test of Mach's Principle and Strong-Field Relativistic Gravity. Found. Phys. Lett., 9, 247-293. doi: 10.1007/BF02186407

Wu, L-A, Bandyopadhyay, S., Sarandy, M. S., \& Lidar, D. A. (2005). Magnetic Susceptibility as a Macroscopic Entanglement Witness. Phys. Rev. A, 72, 032309. doi: 10.1103/PhysRevA.72.032309

Yamasaki, K., Juodkazis, S., Lippert, T., Watanabe, M., Matsuo, S., \& Misawa, H. (2003). Dielectric Breakdown of Rubber Materials by Femtosecond Irradiation. Appl. Phys. A, 76, 325-329. doi: 10.1007/s00339-002-1816-7

Zeilinger, A. (1999). Experiment and the foundations of quantum physics. Rev. Mod. Phys., 71, S288. doi: 10.1103/RevM odPhys.71.S288

\section{Copyrights}

Copyright for this article is retained by the author(s), with first publication rights granted to the journal.

This is an open-access article distributed under the terms and conditions of the Creative Commons Attribution license (http://creativecommons.org/licenses/by/4.0/). 